Pacific Journal of Mathematics

THE STRUCTURE OF TWISTED SU(3) GROUPS 


\title{
THE STRUCTURE OF TWISTED SU(3) GROUPS
}

\section{Albert Jeu-Liang Sheu}

\begin{abstract}
In order to study how the $C^{*}$-algebra $C\left(S_{\mu} U(3)\right)$ of twisted $\mathrm{SU}(3)$ groups introduced by Woronowicz is related to the deformation quantization of the Lie-Poisson $\mathrm{SU}(3)$, we need to understand the algebraic structure of $C\left(S_{\mu} U(3)\right)$ better. In this paper, we shall use Bragiel's result about the irreducible representations of $C\left(S_{\mu} U(3)\right)$ and the theory of groupoid $C^{*}$-algebras to give an explicit description of the $C^{*}$-algebra structure of $C\left(S_{\mu} U(3)\right)$, which indicates that $C\left(S_{\mu} U(3)\right)$ is some kind of foliation $C^{*}$-algebra of the singular symplectic foliation of the Lie-Poisson group $\mathrm{SU}(3)$.
\end{abstract}

In recent years, there has been a rapid growth of interest in the theory of quantum groups [D]. In particular, S. L. Woronowicz has developed a $C^{*}$-algebraic theory of quantum groups, which has motivated a lot of research [B, Po, Ro, S, Va-So, Wo1, Wo2].

In [S], the explicit knowledge of the $C^{*}$-algebra structure of $C\left(S_{\mu} U(2)\right)[\mathbf{W o 1}, \mathbf{S}]$ has helped us to find a deformation quantization [BFFLS, Ri1, Ri2, Ri3] of the Lie-Poisson SU(2) [D, Lu-We], which is in a sense compatible with the quantization of the group structure of $\mathrm{SU}(2)$ by the "twisted groups" $S_{\mu} U(2)$. On the other hand, although both $C\left(S_{\mu} U(2)\right)$ and $C\left(S_{\mu} U(3)\right)$ [Wo1, Wo2] are defined as universal $C^{*}$-algebras of certain generators and relations, the algebraic structure of the latter seems to be much more complicated than that of the former. In [B], Bragiel classified the irreducible representations of the $C^{*}$-algebra $C\left(S_{\mu} U(3)\right)$ of the twisted $\mathrm{SU}(3)$ groups (with $0<\mu<1)$ and showed that $C\left(S_{\mu} U(3)\right)$ is a type-I $C^{*}$-algebra [Pe]. In this paper, enlightened by the ideas in [M-Re, $\mathbf{C u}-\mathbf{M}]$, we shall use Bragiel's result and the theory of groupoid $C^{*}$-algebras [Re] to give an explicit description of the $C^{*}$-algebra structure of $C\left(S_{\mu} U(3)\right)$, which indicates that $C\left(S_{\mu} U(3)\right)$ is some kind of foliation $C^{*}$-algebra of the singular symplectic foliation of the Lie-Poisson group $\mathrm{SU}(3)$ [Co, We, Lu-We].

We shall use freely the concepts and properties of the theory of groupoid $C^{*}$-algebras throughout this paper. A good reference for this is [Re]. First let us fix notations. Let $\mathbb{T}$ be the unit circle in $\mathbb{C}$ and $\mathbb{T}^{2}$ be the two-torus embedded in $\mathbb{C}^{2}$. We shall denote by $\phi$ and 
$\psi$ the two canonical coordinate functions of $\mathbb{T}^{2}$ with values in $\mathbb{T}$. For any groupoid $\mathfrak{G}$, we denote by $\mathfrak{G} \mid P$ the reduction of $\mathfrak{G}$ by the subset $P$ of the unit space of $\mathfrak{G}$ [Re]. If a locally compact group $G$ acts on a space $X$ by an action $\tau$, we shall denote by $X \times{ }_{\tau} G$ the corresponding transformation group groupoid.

We define $\mathfrak{G}:=\overline{\mathbb{Z}}^{3} \times_{\alpha} \mathbb{Z}^{5} \mid \overline{\mathbb{Z}}_{\geq}^{3}$, where $\overline{\mathbb{Z}}=\mathbb{Z} \cup\{+\infty\}$, the subscript $\geq$ denotes the nonnegative part, and $\mathbb{Z}^{5}$ acts on $\overline{\mathbb{Z}}^{3}$ by translation determined by the first three components, i.e. $\alpha(\mu)(\nu)=\nu-\left(\mu_{1}, \mu_{2}, \mu_{3}\right)$ for $\mu \in \mathbb{Z}^{5}$ and $\nu \in \mathbb{Z}^{3}$. Since the last two copies of $\mathbb{Z}$ act trivially on $\overline{\mathbb{Z}}^{3}$, we have $C^{*}(\mathfrak{G}) \cong C^{*}\left(\mathfrak{G}_{0}\right) \otimes C^{*}\left(\mathbb{Z}^{2}\right) \cong C^{*}\left(\mathfrak{G}_{0}\right) \otimes C\left(\mathbb{T}^{2}\right)$, where $\mathfrak{G}_{0}:=\overline{\mathbb{Z}}^{3} \times_{\tau} \mathbb{Z}^{3} \mid \overline{\mathbb{Z}}_{\geq}{ }^{3}$ and $\tau$ is the action by translation. We assume that under the above isomorphism, the standard basis elements $e_{4}$ and $e_{5}$ of $\mathbb{Z}^{5}$ correspond to the conjugates $\bar{\phi}$ and $\bar{\psi}$ of the canonical coordinate functions on $\mathbb{T}^{2}$ (instead of $\phi$ and $\psi$ in order to be more compatible with the notations used in [B] for the later discussion). Recall that the regular representation $\rho_{3}$ of $C^{*}\left(\mathfrak{G}_{0}\right)$ on the open dense invariant subset $\mathbb{Z}_{\geq}^{3}$ is faithful [M-Re], and hence $C^{*}(\mathfrak{G})$ can be faithfully represented on the Hilbert space $l^{2}\left(\mathbb{Z}_{\geq}^{3}\right) \otimes L^{2}\left(\mathbb{T}^{2}\right)$ through $\tilde{\rho}_{3}:=\rho_{3} \otimes m$ where $m$ is the representation of $C\left(\mathbb{T}^{2}\right)$ by multiplication operators on $L^{2}\left(\mathbb{T}^{2}\right)$.

In [B], the irreducible representations of $C\left(S_{\mu} U(3)\right)$ are classified into six 2-parameter families (with parameters in $\mathbb{T}^{2}$ ) of irreducible representations $\pi_{3}, \pi_{21}, \pi_{22}, \pi_{11}, \pi_{12}$ and $\pi_{0}$ (listed here in the same order as in [B]) on Hilbert spaces $l^{2}\left(\mathbb{Z}_{\geq}^{3}\right), l^{2}\left(\mathbb{Z}_{\geq}^{2}\right)$, $l^{2}\left(\mathbb{Z}_{\geq}^{2}\right), l^{2}\left(\mathbb{Z}_{\geq}^{1}\right), l^{2}\left(\mathbb{Z}_{\geq}^{1}\right)$, and $l^{2}\left(\mathbb{Z}_{\geq}^{0}\right)=\mathbb{C}$, respectively. The 2parameter family of irreducible representations $\pi$ (on a Hilbert space $\left.\mathscr{H}_{\pi}\right)$ in the above list determine a representation $\tilde{\pi}$ of $C\left(S_{\mu} U(3)\right)$ on $\mathscr{H}_{\pi} \otimes L^{2}\left(\mathbb{T}^{2}\right)$. Since $\pi_{3}\left(u_{i j}\right)$ 's and $\pi_{3}\left(u_{i j}{ }^{*}\right)$ 's are (finite) linear combinations of weighted (multivariable) shifts on $l^{2}\left(\mathbb{Z}_{\geq}^{3}\right)$ with weight functions extendable to $\overline{\mathbb{Z}}_{\geq}{ }^{3}$ continuously, and since the weight functions involved in each $\pi_{3}\left(u_{i j}\right)$ or $\pi_{3}\left(u_{i j}{ }^{*}\right)$ are products of the canonical functions $\phi, \psi, \bar{\phi}$ and $\bar{\psi}$ on $\mathbb{T}^{2}$ and functions on $\overline{\mathbb{Z}}_{\geq}^{3}$ independent of the parameters in $\mathbb{T}^{2}$, it is easy to identify the 2-parameter family $\tilde{\pi}_{3}\left(u_{i j}\right)$ or $\tilde{\pi}_{3}\left(u_{i j}{ }^{*}\right)$ with an element in $C_{c}(\mathfrak{G}) \subseteq C^{*}(\mathfrak{G})$ (which is faithfully represented on $l^{2}\left(\mathbb{Z}_{\geq}^{3}\right) \otimes L^{2}\left(\mathbb{T}^{2}\right)$ ) for each $u_{i j}$. For example, with $C_{c}\left(\overline{\mathbb{Z}}_{\geq}^{3}\right)$ and $\mathbb{Z}^{5}$ canonically embedded in $C_{c}(\mathfrak{G})$, we have

$$
\begin{array}{ll}
\tilde{\pi}_{3}\left(u_{11}{ }^{*}\right)=e_{1} f_{11}, & \tilde{\pi}_{3}\left(u_{12}{ }^{*}\right)=e_{2} f_{12}, \\
\tilde{\pi}_{3}\left(u_{13}{ }^{*}\right)=e_{5} f_{13}, & \tilde{\pi}_{3}\left(u_{21}{ }^{*}\right)=e_{3} f_{21}, \\
\tilde{\pi}_{3}\left(u_{31}{ }^{*}\right)=e_{4} f_{31}, &
\end{array}
$$


where, for $(N, M, L) \in \overline{\mathbb{Z}}_{\geq}{ }^{3}$,

$$
\begin{aligned}
& f_{11}(N, M, L)=\left(1-\mu^{2(N+1)}\right)^{1 / 2}, \\
& f_{12}(N, M, L)=\mu^{N+1}\left(1-\mu^{2(M+1)}\right)^{1 / 2}, \\
& f_{13}(N, M, L)=\mu^{2+N+M} \\
& f_{21}(N, M, L)=\mu^{N}\left(1-\mu^{2(L+1)}\right)^{1 / 2}, \\
& f_{31}(N, M, L)=\mu^{N+L} .
\end{aligned}
$$

Note that for $0<\mu<1$, the above expressions have canonical meaning even when $N, M$ or $L$ is $\infty$. Thus we can factor the homomorphism $\tilde{\pi}_{3}$ through $C^{*}(\mathfrak{G})$, i.e. there exists a homomorphism

$$
\eta: C\left(S_{\mu} U(3)\right) \rightarrow C^{*}(\mathfrak{G})
$$

such that $\tilde{\pi}_{3}=\tilde{\rho}_{3} \circ \eta$. We shall see later that $\eta$ is in fact injective since all the representations $\tilde{\pi}$ of $C\left(S_{\mu} U(3)\right)$ mentioned above can be factored through $\eta$.

Let us consider the following invariant subsets of the unit space of $\mathfrak{G}$,

$$
\begin{aligned}
X_{3} & =\left\{(N, M, L) \mid N, M, L \in \mathbb{Z}_{\geq}\right\}=\mathbb{Z}_{\geq}{ }^{3}, \\
X_{21} & =\left\{(N, M, L) \mid N, M \in \mathbb{Z}_{\geq} \text {and } L=\infty\right\} \cong \mathbb{Z}_{\geq}{ }^{2}, \\
X_{22} & =\left\{(N, M, L) \mid N, L \in \mathbb{Z}_{\geq} \text {and } M=\infty\right\} \cong \mathbb{Z}_{\geq}{ }^{2}, \\
X_{11} & =\left\{(N, M, L) \mid N \in \mathbb{Z}_{\geq} \text {and } M=L=\infty\right\} \cong \mathbb{Z}_{\geq}, \\
X_{12} & =\left\{(N, M, L) \mid M \in \mathbb{Z}_{\geq} \text {and } N=L=\infty\right\} \cong \mathbb{Z}_{\geq}
\end{aligned}
$$

and $X_{0}=\{(\infty, \infty, \infty)\}$. We define $X_{i}=X_{i 1} \cup X_{i 2}$ for $i=1,2$, and $\sigma_{i}\left(\right.$ resp. $\left.\sigma_{i n}\right)$ to be the quotient map from $C^{*}\left(\mathfrak{G} \mid \bar{X}_{i+1}\right)$ to $C^{*}\left(\mathfrak{G} \mid \bar{X}_{i}\right)$ (resp. $C^{*}\left(\mathfrak{G} \mid \bar{X}_{i n}\right)$ ) for $i=0,1,2$, (resp. $i=1,2$ and $n=1,2$ ) where $\bar{X}_{i}$ is the closure of $X_{i}$ in the unit space of $\mathfrak{G}$. Since $\tilde{\pi}_{3}\left(u_{i j}\right) \tilde{\pi}_{3}\left(u_{i j}{ }^{*}\right)=f_{i j}{ }^{2}$ for the $u_{i j}$ 's listed above and they separate points in $\mathbb{Z}_{\geq} \times \overline{\mathbb{Z}}_{\geq}^{2}$, i.e. points $(N, M, L)$ with $N<\infty$, it is easy to check that $C_{c}\left(X_{3}\right)=C_{c}\left(\mathbb{Z}_{\geq}^{3}\right) \subseteq \operatorname{Im}(\eta)$ (by considering the level sets of these $f_{i j}$ 's). Now since those weights $f_{i j}$ are nonvanishing on $\mathbb{Z}_{\geq}^{3}$ and $C_{c}\left(\mathbb{Z}_{\geq}^{3}\right) \subseteq \operatorname{Im}(\eta)$, the convolution algebra $C_{c}\left(\mathbb{Z}_{\geq}^{3} \times_{\alpha} \mathbb{Z}^{5}\right)$ and hence $C^{*}\left(\mathbb{Z}_{\geq}^{3} \times_{\alpha} \mathbb{Z}^{5}\right) \cong C\left(\mathbb{T}^{2}\right) \otimes \mathscr{K}$ are contained in the $C^{*}$-algebra generated by (the weighted shifts) $\eta\left(u_{i j}{ }^{*}\right)$ of the $u_{i j}{ }^{*}$ 's listed above and hence in $\operatorname{Im}(\eta)$ where $\mathscr{K}$ is the algebra of compact operators (on $l^{2}\left(\mathbb{Z}_{\geq}^{3}\right)$ here).

Now we consider the diagonal homomorphism $\left(\sigma_{21}, \sigma_{22}\right)$ from $C^{*}(\mathfrak{G})$ to $C^{*}\left(\mathfrak{G} \mid \bar{X}_{21}\right) \oplus C^{*}\left(\mathfrak{G} \mid \bar{X}_{22}\right)$. It is easy to see that $\mathfrak{G} \mid \bar{X}_{2 n} \cong$ $\overline{\mathbb{Z}}^{2} \times_{\alpha(2, n)} \mathbb{Z}^{5} \mid \overline{\mathbb{Z}}_{\geq}^{2}$ where $\mathbb{Z}^{5}$ acts on $\overline{\mathbb{Z}}^{2}$ through the action $\alpha(2, n)$ in the way that 2 components (depending on $n$ ) of $\mathbb{Z}^{5}$ act on $\overline{\mathbb{Z}}^{2}$ by 
translation while the other 3 components act trivially. More precisely, $\alpha(2,1)(\mu) \cdot \nu=\nu-\left(\mu_{1}, \mu_{2}\right)$ and $\alpha(2,2)(\mu) \cdot \nu=\nu-\left(\mu_{1}, \mu_{3}\right)$ for $\mu \in \mathbb{Z}^{5}$ and $\nu \in \mathbb{Z}^{2}$. Thus

$C^{*}\left(\mathfrak{G} \mid \bar{X}_{2 n}\right) \cong C^{*}\left(\overline{\mathbb{Z}}^{2} \times_{\tau} \mathbb{Z}^{2} \mid \overline{\mathbb{Z}}_{\geq}^{2}\right) \otimes C^{*}\left(\mathbb{Z}^{3}\right) \cong C^{*}\left(\overline{\mathbb{Z}}^{2} \times_{\tau} \mathbb{Z}^{2} \mid \overline{\mathbb{Z}}_{\geq}^{2}\right) \otimes C\left(\mathbb{T}^{3}\right)$,

where the canonical generators of $\mathbb{Z}^{3}$ are $e_{3}, e_{4}, e_{5}$ when $n=1$, and $e_{2}, e_{4}, e_{5}$ when $n=2$. It is straightforward to check that $\left(\sigma_{21} \circ \eta\right)\left(u_{i j}\right)$ 's $(1 \leq i, j \leq 3)$ are supported in $\overline{\mathbb{Z}}^{2} \times_{\alpha(2,1)} \mathbb{Z}^{4} \mid \overline{\mathbb{Z}}_{\geq}{ }^{2}$ where $\mathbb{Z}^{4}$ is generated by $e_{1}, e_{2}, e_{3}$ and $e_{5}$ in $\mathbb{Z}^{5}$, while $\left(\sigma_{22} \circ \eta\right)\left(u_{i j}\right)$ 's are supported in $\overline{\mathbb{Z}}^{2} \times_{\alpha(2,2)} \mathbb{Z}^{4} \mid \overline{\mathbb{Z}}_{\geq}^{2}$ with $\mathbb{Z}^{4}$ generated by $e_{1}, e_{2}, e_{3}$ and $e_{4}$ in $\mathbb{Z}^{5}$. Furthermore, from the weight functions $f_{i j}$ listed above, it is easy to check that $C_{c}\left(X_{2}\right) \subseteq \operatorname{Im}\left(\sigma_{2} \circ \eta\right)$ and hence

$$
C^{*}\left(\mathbb{Z}^{2} \times_{\alpha(2,1)} \mathbb{Z}^{4} \mid \mathbb{Z}_{\geq}^{2}\right) \oplus C^{*}\left(\mathbb{Z}^{2} \times_{\alpha(2,2)} \mathbb{Z}^{4} \mid \mathbb{Z}_{\geq}^{2}\right) \cong 2 \mathscr{K} \otimes C\left(\mathbb{T}^{2}\right)
$$

is contained in the $C^{*}$-algebra generated by $\left(\sigma_{21}, \sigma_{22}\right)\left(\eta\left(u_{i j}{ }^{*}\right)\right)$ and hence in $\operatorname{Im}\left(\left(\sigma_{21}, \sigma_{22}\right) \circ \eta\right)$. Let $\rho_{2}$ be the faithful regular representation of $\overline{\mathbb{Z}}^{2} \times_{\tau} \mathbb{Z}^{2} \mid \overline{\mathbb{Z}}_{\geq}^{2}$ on $l^{2}\left(\mathbb{Z}_{\geq}^{2}\right)$ and $\tilde{\rho}_{2 n}=\rho_{2} \otimes m$ be the corresponding faithful representation of

$$
C^{*}\left(\overline{\mathbb{Z}}^{2} \times_{\alpha(2, n)} \mathbb{Z}^{4} \mid \overline{\mathbb{Z}}_{\geq}^{2}\right) \cong C^{*}\left(\overline{\mathbb{Z}}^{2} \times_{\tau} \mathbb{Z}^{2} \mid \overline{\mathbb{Z}}_{\geq}^{2}\right) \otimes C\left(\mathbb{T}^{2}\right)
$$

on $l^{2}\left(\mathbb{Z}_{\geq}^{2}\right) \otimes L^{2}\left(\mathbb{T}^{2}\right)$, where the isomorphism identifies $e_{3}, e_{5}$ with $\bar{\phi}, \bar{\psi}$ if $n=1$, and identifies $e_{4}, e_{2}$ with $\bar{\phi}, \bar{\psi}$ if $n=2$. Then it can be easily checked that

$$
\tilde{\rho}_{2 n}\left(\sigma_{2 n}\left(\eta\left(u_{i j}\right)\right)\right)=\tilde{\pi}_{2 n}\left(u_{i j}\right)
$$

(note that in the above identification, the symbols $N$ and $M$ used in [B] need be interchanged when $n=2$ ) and hence $\tilde{\pi}_{2 n}$ factors through $\eta$. Let $\eta_{2 n}:=\sigma_{2 n} \circ \eta$.

Now we consider $\sigma_{12} \circ \sigma_{2}$ and $\sigma_{11} \circ \sigma_{2}$. Since clearly $\sigma_{12} \circ \sigma_{2}$ factors through $\sigma_{21}$ and $\sigma_{11} \circ \sigma_{2}$ factors through $\sigma_{21}$ and $\sigma_{22}$, we may talk about $\sigma_{12} \circ \sigma_{21}\left(=\sigma_{12} \circ \sigma_{2}\right)$ and $\sigma_{11} \circ \sigma_{21}=\sigma_{11} \circ \sigma_{22}\left(=\sigma_{11} \circ \sigma_{2}\right)$ by abuse of language. Note that

$$
C^{*}\left(\mathbb{Z}^{2} \times_{\alpha(2,1)} \mathbb{Z}^{4} \mid \mathbb{Z}_{\geq}^{2}\right) \oplus C^{*}\left(\mathbb{Z}^{2} \times_{\alpha(2,2)} \mathbb{Z}^{4} \mid \mathbb{Z}_{\geq}{ }^{2}\right) \subseteq C^{*}\left(\mathfrak{G} \mid X_{2}\right) \subseteq \operatorname{ker}\left(\sigma_{1 n}\right)
$$

because $\left(\mathbb{Z}^{2} \times_{\alpha(2,1)} \mathbb{Z}^{4} \mid \mathbb{Z}_{\geq}^{2}\right) \cup\left(\mathbb{Z}^{2} \times_{\alpha(2,2)} \mathbb{Z}^{4} \mid \mathbb{Z}_{\geq}{ }^{2}\right) \subseteq X_{2}$. It is again easy to see that $\mathfrak{G}\left|\bar{X}_{1 n} \cong \overline{\mathbb{Z}} \times_{\alpha(1, n)} \mathbb{Z}^{5}\right| \overline{\mathbb{Z}}_{\geq}$where $\mathbb{Z}^{5}$ acts on $\overline{\mathbb{Z}}$ through the action $\alpha(1, n)$ in the way that one component (depending on $n$ ) of $\mathbb{Z}^{5}$ act on $\overline{\mathbb{Z}}$ by translation while the other 4 components act trivially. 
More precisely, $\alpha(1,1)(\mu) \cdot \nu=\nu-\mu_{1}$ and $\alpha(1,2)(\mu) \cdot \nu=\nu-\mu_{2}$ for $\mu \in \mathbb{Z}^{5}$ and $\nu \in \mathbb{Z}$. Thus

$$
C^{*}\left(\mathfrak{G} \mid \bar{X}_{1 n}\right) \cong C^{*}\left(\overline{\mathbb{Z}} \times_{\tau} \mathbb{Z} \mid \overline{\mathbb{Z}}_{\geq}\right) \otimes C^{*}\left(\mathbb{Z}^{4}\right) \cong C^{*}\left(\overline{\mathbb{Z}} \times_{\tau} \mathbb{Z} \mid \overline{\mathbb{Z}}_{\geq}\right) \otimes C\left(\mathbb{T}^{4}\right),
$$

where the canonical generators of $\mathbb{Z}^{4}$ are $e_{2}, e_{3}, e_{4}, e_{5}$ when $n=1$, and $e_{1}, e_{3}, e_{4}, e_{5}$ when $n=2$. It is straightforward to check that $\left(\sigma_{11} \circ \sigma_{2} \circ \eta\right)\left(u_{i j}\right)$ 's $(1 \leq i, j \leq 3)$ are supported in $\overline{\mathbb{Z}} \times_{\alpha(1,1)} \mathbb{Z}^{3} \mid \overline{\mathbb{Z}}_{\geq}$ where $\mathbb{Z}^{3}$ is generated by $e_{1}, e_{2}$ and $e_{3}$ in $\mathbb{Z}^{5}$, while the $\left(\sigma_{12} \circ \sigma_{2} \circ \eta\right)\left(u_{i j}\right)$ 's are supported in $\overline{\mathbb{Z}} \times_{\alpha(1,2)} \mathbb{Z}^{4} \mid \overline{\mathbb{Z}}_{\geq}$with $\mathbb{Z}^{4}$ generated by $e_{1}, e_{2}, e_{3}$ and $e_{5}$ in $\mathbb{Z}^{5}$. Let $\rho_{1}$ be the faithful regular representation of $\overline{\mathbb{Z}} \times_{\tau} \mathbb{Z} \mid \overline{\mathbb{Z}}_{\geq}$on $l^{2}\left(\mathbb{Z}_{\geq}\right)$and $\tilde{\rho}_{11}=\rho_{1} \otimes m$ be the corresponding faithful representation of

$$
C^{*}\left(\overline{\mathbb{Z}} \times_{\alpha(1,1)} \mathbb{Z}^{3} \mid \overline{\mathbb{Z}}_{\geq}\right) \cong C^{*}\left(\overline{\mathbb{Z}} \times_{\tau} \mathbb{Z} \mid \overline{\mathbb{Z}}_{\geq}\right) \otimes C\left(\mathbb{T}^{2}\right)
$$

on $l^{2}\left(\mathbb{Z}_{\geq}^{2}\right) \otimes L^{2}\left(\mathbb{T}^{2}\right)$, where the isomorphism identifies $e_{3}$ and $e_{2}$ with $\bar{\phi}$ and $\bar{\psi}$ respectively. Then it can be easily checked that

$$
\tilde{\rho}_{11}\left(\left(\sigma_{11} \circ \sigma_{2} \circ \eta\right)\left(u_{i j}\right)\right)=\tilde{\pi}_{11}\left(u_{i j}\right)
$$

and hence $\tilde{\pi}_{11}$ factors through $\eta$ and $\eta_{11}:=\sigma_{11} \circ \sigma_{2} \circ \eta=\sigma_{11} \circ \eta_{21}=$ $\sigma_{11} \circ \eta_{22}$. On the other hand, we have

$$
C^{*}\left(\overline{\mathbb{Z}} \times_{\alpha(1,2)} \mathbb{Z}^{4} \mid \overline{\mathbb{Z}}_{\geq}\right) \cong C^{*}\left(\overline{\mathbb{Z}} \times_{\tau} \mathbb{Z} \mid \overline{\mathbb{Z}} \geq\right) \otimes C\left(\mathbb{T}^{3}\right),
$$

where the conjugates of the three canonical coordinate functions of $\mathbb{T}^{3}$ correspond to the generators $e_{1}, e_{3}$ and $e_{5}$ in $\mathbb{Z}^{5}$. Composing the above identification with id $\otimes \kappa_{12}$, we get a homomorphism $\lambda_{12}$ from $C^{*}\left(\overline{\mathbb{Z}} \times_{\alpha(1,2)} \mathbb{Z}^{4} \mid \overline{\mathbb{Z}}_{\geq}\right)$to $C^{*}\left(\overline{\mathbb{Z}} \times_{\tau} \mathbb{Z} \mid \overline{\mathbb{Z}}_{\geq}\right) \otimes C\left(\mathbb{T}^{2}\right)$, where $\kappa_{12}$ is the homomorphism from $C\left(\mathbb{T}^{3}\right)$ to $C\left(\mathbb{T}^{2}\right)$ induced by the map from $\mathbb{T}^{2}$ to $\mathbb{T}^{3}$ sending $z \in \mathbb{T}^{2}$ to $\left(z_{1},-z_{1}, z_{2}\right)$. Let $\tilde{\rho}_{12}=\rho_{1} \otimes m$ be the faithful representation of $C^{*}\left(\overline{\mathbb{Z}} \times_{\tau} \mathbb{Z} \mid \overline{\mathbb{Z}} \geq\right) \otimes C\left(\mathbb{T}^{2}\right) \supseteq \operatorname{Im}\left(\eta_{12}\right)$, where $\eta_{12}=\lambda_{12} \circ\left(\sigma_{12} \circ \sigma_{2} \circ \eta\right)=\lambda_{12} \circ\left(\sigma_{12} \circ \sigma_{21} \circ \eta\right)$. (Here we use the convention that $f \circ g$ is meaningful whenever $\operatorname{Im}(g) \subseteq \operatorname{Dom}(f)$.) Then $\tilde{\rho}_{12} \circ \lambda_{12}$ defines a representation of $\operatorname{Im}\left(\sigma_{12} \circ \sigma_{2} \circ \eta\right)$ on $l^{2}\left(\mathbb{Z}_{\geq}\right) \otimes L^{2}\left(\mathbb{T}^{2}\right)$. It is straightforward to check that

$$
\left(\tilde{\rho}_{12} \circ \lambda_{12}\right)\left(\left(\sigma_{12} \circ \sigma_{2} \circ \eta\right)\left(u_{i j}\right)\right)=\tilde{\pi}_{12}\left(u_{i j}\right)
$$

(note that in [B], $M$ is replaced by $N$ ) for all $i, j$. From the weight functions $f_{i j}$ listed above, it is easy to check that $C_{c}\left(X_{1}\right) \subseteq$ $\operatorname{Im}\left(\sigma_{1} \circ \sigma_{2} \circ \eta\right)$. So by the formulas for $\pi_{1 n}\left(u_{i j}\right)$ in [B], it is not hard to see that

$$
\begin{aligned}
& C^{*}\left(\mathbb{Z} \times_{\alpha(1,1)}\right.\left.\mathbb{Z}^{3} \mid \mathbb{Z}_{\geq}\right) \oplus \lambda_{12}\left(C^{*}\left(\mathbb{Z} \times_{\alpha(1,2)} \mathbb{Z}^{4} \mid \mathbb{Z}_{\geq}\right)\right) \\
& \cong 2 C^{*}\left(\mathbb{Z} \times{ }_{\tau} \mathbb{Z} \mid \mathbb{Z}_{\geq}\right) \otimes C\left(\mathbb{T}^{2}\right) \cong 2 \mathscr{K} \otimes C\left(\mathbb{T}^{2}\right)
\end{aligned}
$$


is contained in the $C^{*}$-algebra generated by $\left(\eta_{11}, \eta_{12}\right)\left(u_{i j}{ }^{*}\right)$ and hence in $\operatorname{Im}\left(\left(\eta_{11}, \eta_{12}\right)\right)$. Notice that

$$
C^{*}\left(\mathbb{Z} \times_{\alpha(1,1)} \mathbb{Z}^{3} \mid \mathbb{Z}_{\geq}\right) \oplus C^{*}\left(\mathbb{Z} \times_{\alpha(1,2)} \mathbb{Z}^{4} \mid \mathbb{Z}_{\geq}\right) \subseteq C^{*}\left(\mathfrak{G} \mid X_{1}\right)
$$

is contained in the kernel of $\sigma_{0}$.

Now we consider $\sigma_{0} \circ \sigma_{1} \circ \sigma_{2}$. Since $\sigma_{0} \circ \sigma_{1} \circ \sigma_{2}$ clearly factors through $\sigma_{11} \circ \sigma_{2}$ and $\sigma_{12} \circ \sigma_{2}$, we may talk about $\sigma_{0} \circ \sigma_{11} \circ \sigma_{2}=$ $\sigma_{0} \circ \sigma_{12} \circ \sigma_{2}=\sigma_{0} \circ \sigma_{1} \circ \sigma_{2}$ by abuse of language. Note that $C^{*}\left(\mathfrak{G} \mid X_{0}\right)=$ $C^{*}\left(\mathbb{Z}^{5}\right) \cong C\left(\mathbb{T}^{5}\right)$ and that $\left(\sigma_{0} \circ \sigma_{1} \circ \sigma_{2} \circ \eta\right)\left(u_{i j}\right)$ 's $(1 \leq i, j \leq 3)$ are supported in $\mathbb{Z}^{3}$ generated by $e_{1}, e_{2}$ and $e_{3}$ in $\mathbb{Z}^{5}$. Composing the identification $C^{*}\left(\mathbb{Z}^{3}\right) \cong C\left(\mathbb{T}^{3}\right)$ with $\kappa_{0}$ (where the generators $e_{1}, e_{2}$, $e_{3}$ are identified with the conjugates of the corresponding coordinate functions of $\mathbb{T}^{3}$ ), we get a homomorphism $\lambda_{0}$ from $C^{*}\left(\mathbb{Z}^{3}\right)$ to $C\left(\mathbb{T}^{2}\right)$, where $\kappa_{0}$ is the homomorphism from $C\left(\mathbb{T}^{3}\right)$ to $C\left(\mathbb{T}^{2}\right)$ induced by the map from $\mathbb{T}^{2}$ to $\mathbb{T}^{3}$ sending $z \in \mathbb{T}^{2}$ to $\left(z_{1}, z_{2},-z_{1}\right)$. Let $\tilde{\rho}_{0}:=$ $m$. Then $\tilde{\rho} \circ \lambda_{0}$ is a representation of $C^{*}\left(\mathbb{Z}^{3}\right)$ on $L^{2}\left(\mathbb{T}^{2}\right)$. It is straightforward to check that

$$
\left(\tilde{\rho}_{0} \circ \eta_{0}\right)\left(u_{i j}\right)=\tilde{\pi}_{0}\left(u_{i j}\right)
$$

for all $i, j$, where $\eta_{0}=\lambda_{0} \circ \sigma_{0} \circ \sigma_{1} \circ \sigma_{2} \circ \eta$ is a homomorphism from $C\left(S_{\mu} U(3)\right)$ to $C^{*}\left(\mathbb{Z}^{2}\right) \cong C\left(\mathbb{T}^{2}\right)$. Comparing the definitions of $\kappa_{12}$ and $\kappa_{0}$ and relating the generators of their domains $C^{*}\left(\mathbb{Z}^{3}\right)$ to those of $\mathbb{Z}^{5}$ as we specified above, it is easy to check that $\eta_{0}$ factors through $\eta_{11}$ and $\eta_{12}$, say $\eta_{0}=\tilde{\omega}_{0} \circ\left(\eta_{11}, \eta_{12}\right)$ for some $\tilde{\omega}_{0}$ defined on $\operatorname{Im}\left(\eta_{11}, \eta_{12}\right)$. Note that $\operatorname{ker}\left(\tilde{\omega}_{0}\right)$ contains the subalgebra

$$
C^{*}\left(\mathbb{Z} \times_{\alpha(1,1)} \mathbb{Z}^{3} \mid \mathbb{Z}_{\geq}\right) \oplus \lambda_{12}\left(C^{*}\left(\mathbb{Z} \times_{\alpha(1,2)} \mathbb{Z}^{4} \mid \mathbb{Z}_{\geq}\right)\right) \cong 2 \mathscr{K} \otimes C\left(\mathbb{T}^{2}\right) .
$$

Now we summarize what we have so far. There are homomorphisms $\eta_{3}=\eta, \eta_{21}, \eta_{22}, \eta_{11}, \eta_{12}$ and $\eta_{0}$ from $C\left(S_{\mu} U(3)\right)$ to

$$
\begin{gathered}
C^{*}(\mathfrak{G})=C^{*}\left(\overline{\mathbb{Z}}^{3} \times_{\alpha} \mathbb{Z}^{5} \mid \overline{\mathbb{Z}}_{\geq}^{3}\right)=C\left(\overline{\mathbb{Z}}^{3} \times_{\tau} \mathbb{Z}^{3} \mid \overline{\mathbb{Z}}_{\geq}^{3}\right) \otimes C\left(\mathbb{T}^{2}\right), \\
C^{*}\left(\overline{\mathbb{Z}}^{2} \times_{\tau} \mathbb{Z}^{2} \mid \overline{\mathbb{Z}}_{\geq}{ }^{2}\right) \otimes C\left(\mathbb{T}^{2}\right), \quad C^{*}\left(\overline{\mathbb{Z}}^{2} \times_{\tau} \mathbb{Z}^{2} \mid \overline{\mathbb{Z}}_{\geq}{ }^{2}\right) \otimes C\left(\mathbb{T}^{2}\right), \\
C^{*}\left(\overline{\mathbb{Z}} \times_{\tau} \mathbb{Z} \mid \overline{\mathbb{Z}}_{\geq}\right) \otimes C\left(\mathbb{T}^{2}\right), \quad C^{*}\left(\overline{\mathbb{Z}} \times_{\tau} \mathbb{Z} \mid \overline{\mathbb{Z}}_{\geq}\right) \otimes C\left(\mathbb{T}^{2}\right) \quad \text { and } \quad C\left(\mathbb{T}^{2}\right),
\end{gathered}
$$

respectively, such that

(1) each $\eta_{i}$ or $\eta_{i n}$ factors through $\eta_{j}$ with $j>i$, where $\eta_{i}:=$ $\left(\eta_{i 1}, \eta_{i 2}\right)$ if $i=1,2$. In fact, $\eta_{21}=\omega_{21} \circ \eta, \eta_{22}=\omega_{22} \circ \eta, \eta_{11}=$ $\omega_{11} \circ \eta_{21}, \eta_{11}=\omega_{11}^{\prime} \circ \eta_{22}, \eta_{12}=\omega_{12} \circ \eta_{21}, \eta_{0}=\omega_{0} \circ \eta_{11}$ and $\eta_{0}=\omega_{0}^{\prime} \circ \eta_{12}$ for some $\omega$ 's defined on the range of the corresponding $\eta$ 's. 
(2) Let $\eta_{i}=\tilde{\omega}_{i} \circ \eta_{i+1}$ for a suitable homomorphism $\tilde{\omega}_{i}$ defined on $\operatorname{Im}\left(\eta_{i+1}\right)$. Then $\operatorname{ker}\left(\tilde{\omega}_{i}\right)$ contains a copy of $C\left(\mathbb{T}^{2}\right) \otimes \mathscr{K}$ if $i=2$, and contains two copies of $C\left(\mathbb{T}^{2}\right) \otimes \mathscr{K}$ if $i=0$ or 1 . Furthermore, $\operatorname{Im}\left(\eta_{0}\right) \cong C\left(\mathbb{T}^{2}\right)$. Note that $\operatorname{Ker}\left(\eta_{i}\right)=\eta_{i+1}^{-1}\left(\operatorname{Ker}\left(\tilde{\omega}_{i}\right)\right)$.

(3) $\tilde{\pi}_{i}=\tilde{\rho}_{i} \circ \eta_{i} \quad(i=0,3)$ and $\tilde{\pi}_{i n}=\tilde{\rho}_{i n} \circ \eta_{i n}(i=1,2)$ for some faithful representations $\tilde{\rho}_{i}$ and $\tilde{\rho}_{i n}$ on $\operatorname{Im}\left(\eta_{i}\right)$ and $\operatorname{Im}\left(\eta_{i n}\right)$ respectively. Since the irreducible representations of $C\left(S_{\mu} U(3)\right)$ are classified by those 2-parameter families of $\pi_{0}, \pi_{11}, \pi_{12}, \pi_{21}, \pi_{22}$, and $\pi_{3}$, the spectrum of $C\left(S_{\mu} U(3)\right)$ is a disjoint union of 6 copies of $\mathbb{T}^{2}$ as a set. On the other hand, by (1)-(3), all these representations $\pi_{i}$ 's (or $\pi_{i n}$ 's) factor through $\eta_{j}$ (or $\eta_{j n}$ ) with $j>i$ and hence $\eta=\eta_{3}$ is faithful. Thus, the type I $C^{*}$-algebra $C\left(S_{\mu} U(3)\right)$ has a composition sequence

$$
0 \subseteq \mathscr{I}_{3}=\operatorname{Ker}\left(\eta_{2}\right) \subseteq \mathscr{I}_{2}=\operatorname{Ker}\left(\eta_{1}\right) \subseteq \mathscr{I}_{1}=\operatorname{Ker}\left(\eta_{0}\right) \subseteq \mathscr{I}_{0}=C\left(S_{\mu} U(3)\right)
$$

such that $\mathscr{I}_{3}=\operatorname{Ker}\left(\tilde{\omega}_{2}\right), \mathscr{I}_{2} / \mathscr{I}_{3} \cong \operatorname{Ker}\left(\tilde{\omega}_{1}\right), \mathscr{I}_{1} / \mathscr{I}_{2} \cong \operatorname{Ker}\left(\tilde{\omega}_{0}\right)$ and $\mathscr{I}_{0} / \mathscr{I}_{1} \cong \operatorname{Im}\left(\eta_{0}\right) \cong C\left(\mathbb{T}^{2}\right)$. Note that $C\left(Y_{i+1}\right) \otimes \mathscr{K}(\mathscr{H}) \subseteq \operatorname{Ker}\left(\tilde{\omega}_{i}\right) \subseteq$ $\operatorname{Im}\left(\eta_{i+1}\right) \subseteq C\left(Y_{i+1}\right) \otimes \mathscr{B}(\mathscr{H})$ (for some $L^{2}$-space $\left.\mathscr{H}\right)$, where $Y_{k}$ is homeomorphic to $\mathbb{T}^{2}$ if $k=3$ or 0 , and to the disjoint union of 2 copies of $\mathbb{T}^{2}$ if $k=2$ or 1 . If $C\left(Y_{i+1}\right) \otimes \mathscr{K}(\mathscr{H}) \neq \operatorname{Ker}\left(\tilde{\omega}_{i}\right)$, then we have non-trivial irreducible representations of $\operatorname{Ker}\left(\tilde{\omega}_{i}\right) / C\left(Y_{i+1}\right) \otimes$ $\mathscr{K}(\mathscr{H})$ which will induce irreducible representations of $C\left(S_{\mu} U(3)\right)$ not unitarily equivalent to any of the $\pi$ 's found in [B]. So we have $C\left(Y_{i+1}\right) \otimes \mathscr{K}(\mathscr{H})=\operatorname{Ker}\left(\tilde{\omega}_{i}\right)$.

We summarize what we obtained about the structure of the $C^{*}$ algebra $C\left(S_{\mu} U(3)\right)$ in the following theorem.

THEOREM. The $C^{*}$-algebra $C\left(S_{\mu} U(3)\right)$ of the twisted $\mathrm{SU}(3)$ group has the composition sequence

$$
\mathscr{I}_{3} \subseteq \mathscr{I}_{2} \subseteq \mathscr{I}_{1} \subseteq \mathscr{I}_{0}=C\left(S_{\mu} U(3)\right)
$$

such that

$$
\mathscr{I}_{0} / \mathscr{I}_{1} \cong C\left(\mathbb{T}^{2}\right), \quad \mathscr{I}_{1} / \mathscr{I}_{2} \cong \mathscr{I}_{2} / \mathscr{I}_{3} \cong 2 C\left(\mathbb{T}^{2}\right) \otimes \mathscr{K}
$$

and $\mathscr{I}_{3} \cong C\left(\mathbb{T}^{2}\right) \otimes \mathscr{K}$.

We remark that the above decomposition of $C\left(S_{\mu} U(3)\right)$ is compatible with the singular foliation of the Lie-Poisson SU(3) [Lu-We] by the symplectic leaves [We]. More precisely, there are six 2-parameter families (with parameters in $\mathbb{T}^{2}$ ) of symplectic leaves diffeomorphic to $\mathbb{C}^{0}, \mathbb{C}^{1}, \mathbb{C}^{1}, \mathbb{C}^{2}, \mathbb{C}^{2}$ and $\mathbb{C}^{3}$, respectively as pointed out by 
A. Weinstein in a private communication. With each leaf of positive dimension quantized by the Weyl quantization [Hö, Vo], it is likely that we can find a deformation quantization (in the sense of [Ri1]) of the Poisson SU(3) as we did for the case of Poisson SU(2) in [S]. In a sense as explained in [S], $C\left(S_{\mu} U(3)\right)$ can be regarded as a foliation $C^{*}$-algebra of the (singular) symplectic foliation on $\mathrm{SU}(3)$.

With some more effort to analyse the data obtained, we are able to describe the topology of the spectrum $Y$ of $C\left(S_{\mu} U(3)\right)$. In order to do so, we shall say that a copy of $\mathbb{T}^{2}$ approximates another copy of $\mathbb{T}^{2}$ in a topological space in type ... if any sequence in the first $\mathbb{T}^{2}$ converges to any element in the second $\mathbb{T}^{2}$, and in type - - $>$ or $=$, if a sequence $z(n)$ in the first $\mathbb{T}^{2}$ converges to $w$ in the second $\mathbb{T}^{2}$ if and only if $z(n)_{2} \rightarrow w_{2}, z(n)_{1} \rightarrow w_{1}$, $z(n)_{1} z(n)_{2} \rightarrow \bar{w}_{2}$ or $z(n)_{1} z(n)_{2} \rightarrow w_{1} w_{2}$ respectively. Now clearly $Y$ is a union of the above $Y_{k}$ 's, and by a more detailed analysis of the factorizability among $\eta$ 's than the one specified in (1), we can conclude that $Y$ is a disjoint union of $Y_{0}, Y_{11}, Y_{12}, Y_{21}, Y_{22}$ and $Y_{3}$ (each homeomorphic to $\mathbb{T}^{2}$ ) such that (i) $Y_{3}$ is open dense in $Y$ in the way that $Y_{3}$ approximates $Y_{21}, Y_{22}, Y_{11}, Y_{12}$ and $Y_{0}$ in type $\ldots,-, \ldots, \ldots$, and ..., respectively, (ii) $Y_{21}$ and $Y_{22}$ are disjoint open sets with dense union $Y_{2}=Y_{21} \cup Y_{22}$ in $Y \backslash Y_{3}$ such that $Y_{21}$ approximates $Y_{11}, Y_{12}$, and $Y_{0}$ in type - , and ... respectively, and $Y_{22}$ approximates $Y_{11} Y_{12}$ and $Y_{0}$ in type and ... respectively $\left(Y_{12} \cap \bar{Y}_{22}=\varnothing\right)$, (iii) $Y_{11}$ and $Y_{12}$ are disjoint open sets with dense union $Y_{1}=Y_{11} \cup Y_{12}$ in $Y \backslash\left(Y_{3} \cup Y_{2}\right)$ such that $Y_{11}$ and $Y_{12}$ approximating $Y_{0}$ in type $=$ and - respectively.

\section{REFERENCES}

[BFFLS] F. Bayen, M. Flato, C. Fronsdal, A. Lichnerowicz and D. Sternheimer, Deformation theory and quantization, I, II, Ann. Physics, 110 (1978), 61110, 111-151.

[B] K. Bragiel, The twisted SU(3) group, Irreducible *-representations of the $C^{*}$-algebra $C\left(S_{\mu} U(3)\right)$, Letters in Math. Phys., 17 (1989), 37-44.

[Co] A. Connes, $A$ survey of foliation and operator algebras, Proc. Symp. Pure Math., Vol. 38, Part I, Amer. Math. Soc., Providence, R.I. (1982), 521-628?

[Cu-M] R. E. Curto and P. S. Muhly, $C^{*}$-algebras of multiplication operators on Bergman spaces, J. Funct. Anal., 64 (1985), 315-329.

[D] V. G. Drinfeld, Quantum groups, Proc. I.C.M. Berkeley 1986, Vol. 1, 789820, Amer. Math. Soc., Providence, R.I. 1987.

[Hö] L. Hörmander, The Weyl calculus of pseudo-differential operators, Comm. Pure Appl. Math., 32 (1979), 359-443. 
[Lu-We] J. H. Lu and A. Weinstein, Poisson Lie groups, dressing transformations and Bruhat decompositions, J. Differential Geom., 31 (1990), 501-526.

[M-Re] P. S. Muhly and J. N. Renault, C*-algebra of multivariable Wiener-Hopf operators, Trans. Amer. Math. Soc., 274 (1982), 1-44.

[Pe] G. K. Pedersen, $C^{*}$-algebras and their Automorphism Groups, Academic Press, New York, 1979.

[Po] P. Podles, Quantum spheres, Letters in Math. Phys., 14 (1987), 193-202.

[Re] J. Renault, A Groupoid Approach to $C^{*}$-Algebras, Lecture Notes in Mathematics, Vol. 793, Springer-Verlag, New York, 1980.

[Ri1] M. A. Rieffel, Deformation quantization and operator algebras, preprint.

[Ri2] - Deformation quantization of Heisenberg manifolds, Comm. Math. Phys., 122 (1989), 531-562.

[Ri3] _ Lie group convolution algebras as deformation quantization of linear Poisson structures, Amer. J. Math., 112 (1990), 657-686.

[Ro] M. Rosso, Comparaison des groupes SU(2) quantiques de Drinfeld et de Woronowicz, C. R. Acad. Sci. Paris, 304 (1987), 323-326.

[S] A. J. L. Sheu, Quantization of the Poisson SU(2) and its Poisson homogeneous space-the 2-sphere, Comm. Math. Phys., 135 (1991), 217-232.

[Va-So] L. L. Vaksman and Ya. S. Soibelman, Function algebra on quantum group SU(2), Funktsional. Anal. i Prilozhen., 22 (1988), No. 2, 1-14 (in Russian).

[Vo] A. Voros, An algebra of pseudodifferential operators and the asymptotics of quantum mechanics, J. Funct. Anal., 29 (1978), 104-132.

[We] A. Weinstein, The local structure of Poisson manifolds, J. Differential Geom., 18 (1983), 523-557.

[Wo1] S. L. Woronowicz, Twisted SU(2) group: an example of a non-commutative differential calculus, Publ. RIMS, 23 (1987), 117-181.

[Wo2] — Compact matrix pseudogroups, Comm. Math. Phys., 111 (1987), 613-665.

Received July 10, 1990. Partially supported by NSF-Grant DMS-9002895. The result of this paper was presented at the AMS-IMS-SIAM Joint Summer Research Conference, June 18, 1990, at Amherst, Massachusetts, U.S.A.

UNIVERSITY OF KANSAS

LAWRENCE, KS 66045-2142 



\section{PACIFIC JOURNAL OF MATHEMATICS EDITORS}

\author{
V. S. VARADARAJAN \\ (Managing Editor) \\ University of California \\ Los Angeles, CA 90024-1555-05 \\ Herbert Clemens \\ University of Utah \\ Salt Lake City, UT 84112 \\ THOMAS ENRIGHT \\ University of California, San Diego \\ La Jolla, CA 92093
}

Nicholas ERcolani

University of Arizona

Tucson, AZ 85721

R. FINN

Stanford University

Stanford, CA 94305

VAUGHAN F. R. JONES

University of California

Berkeley, CA 94720

STEVEN KeRCKHOFF

Stanford University

Stanford, CA 94305

\section{C. MOORE \\ University of California \\ Berkeley, CA 94720}

MARTIN SChaRLEMANN

University of California

Santa Barbara, CA 93106

HAROLd STARK

University of California, San Diego

La Jolla, CA 92093

\section{ASSOCIATE EDITORS}

\begin{tabular}{|c|c|c|c|c|}
\hline R. ARENS & $\begin{array}{l}\text { E. F. BECKENBACH } \\
(1906-1982)\end{array}$ & NeumanN & $\begin{array}{l}\text { F. WoLF } \\
(1904-1989)\end{array}$ & K. YoshidA \\
\hline \multicolumn{5}{|c|}{ SUPPORTING INSTITUTIONS } \\
\hline \multirow{2}{*}{\multicolumn{2}{|c|}{$\begin{array}{l}\text { IVERSITY OF ARIZONA } \\
\text { IVERSITY OF BRITISH COLUMBIA }\end{array}$}} & UNIVERS & Y OF OREGON & \\
\hline & & UNIVERS & OF SOUTHER & RNIA \\
\hline \multicolumn{2}{|c|}{ IFORNIA INSTITUTE OF TECHNOLOGY } & STANFOI & UNIVERSITY & \\
\hline \multirow{2}{*}{\multicolumn{2}{|c|}{$\begin{array}{l}\text { IVERSITY OF CALIFORNIA } \\
\text { NTANA STATE UNIVERSITY }\end{array}$}} & UNIVERS & OF HAWAII & \\
\hline \multirow{2}{*}{\multicolumn{2}{|c|}{$\begin{array}{l}\text { NTANA STATE UNIVERSITY } \\
\text { IVERSITY OF NEVADA, RENO }\end{array}$}} & UNIVERS & Y OF TOKYO & \\
\hline & & UNIVERS & OF UTAH & \\
\hline \multirow{2}{*}{\multicolumn{2}{|c|}{$\begin{array}{l}\text { N MEXICO STATE UNIVERSITY } \\
\text { EGON STATE UNIVERSITY }\end{array}$}} & WASHING & N STATE UNI & RSITY \\
\hline & & UNIVERS & OF WASHING & \\
\hline
\end{tabular}

The Supporting Institutions listed above contribute to the cost of publication of this Journal, but they are not owners or publishers and have no responsibility for its content or policies.

Mathematical papers intended for publication in the Pacific Journal of Mathematics should be in typed form or offset-reproduced (not dittoed), double spaced with large margins. Please do not use built up fractions in the text of the manuscript. However, you may use them in the displayed equations. Underline Greek letters in red, German in green, and script in blue. The first paragraph must be capable of being used separately as a synopsis of the entire paper. In particular it should contain no bibliographic references. Please propose a heading for the odd numbered pages of less than 35 characters. Manuscripts, in triplicate, may be sent to any one of the editors. Please classify according to the 1991 Mathematics Subject Classification scheme which can be found in the December index volumes of Mathematical Reviews. Supply name and address of author to whom proofs should be sent. All other communications should be addressed to the managing editor, or Elaine Barth, University of California, Los Angeles, California 90024-1555-05.

There are page-charges associated with articles appearing in the Pacific Journal of Mathematics. These charges are expected to be paid by the author's University, Government Agency or Company. If the author or authors do not have access to such Institutional support these charges are waived. Single authors will receive 50 free reprints; joint authors will receive a total of 100 free reprints. Additional copies may be obtained at cost in multiples of 50 .

The Pacific Journal of Mathematics (ISSN 0030-8730) is published monthly except for July and August. Regular subscription rate: $\$ 190.00$ a year (10 issues). Special rate: $\$ 95.00$ a year to individual members of supporting institutions.

Subscriptions, orders for numbers issued in the last three calendar years, and changes of address should be sent to Pacific Journal of Mathematics, P.O. Box 969, Carmel Valley, CA 93924, U.S.A. Old back numbers obtainable from Kraus Periodicals Co., Route 100, Millwood, NY 10546.

The Pacific Journal of Mathematics at P.O. Box 969, Carmel Valley, CA 93924 (ISSN 0030-8730) is published monthly except for July and August. Second-class postage paid at Carmel Valley, Californiä 93924, and additional mailing offices. Postmaster: send address changes to Pacific Journal of Mathematics, P.O. Box 969, Carmel Valley, CA 93924.

\section{PUBLISHED BY PACIFIC JOURNAL OF MATHEMATICS, A NON-PROFIT CORPORATION}




\section{Pacific Journal of Mathematics}

\section{Vol. 151, No. $2 \quad$ December, 1991}

Michael G. Eastwood and A. M. Pilato, On the density of twistor

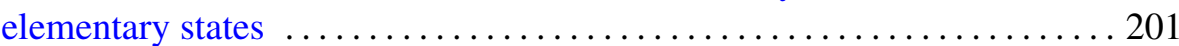

Brian E. Forrest, Arens regularity and discrete groups $\ldots \ldots \ldots \ldots \ldots \ldots 217$

Yu Li Fu, On Lipschitz stability for F.D.E ..................... 229

Douglas Austin Hensley, The largest digit in the continued fraction expansion of a rational number $\ldots \ldots \ldots \ldots \ldots \ldots \ldots \ldots \ldots \ldots \ldots . \ldots 237$

Uwe Kaiser, Link homotopy in $\mathbb{R}^{3}$ and $S^{3}$

Ronald Leslie Lipsman, The Penney-Fujiwara Plancherel formula for abelian symmetric spaces and completely solvable homogeneous

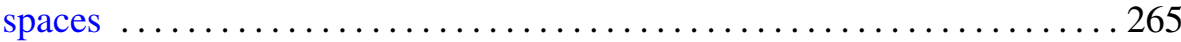

Florin G. Radulescu, Singularity of the radial subalgebra of $\mathscr{L}\left(F_{N}\right)$ and the

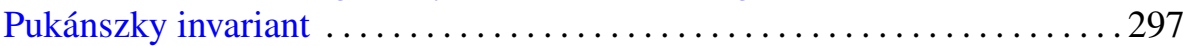

Albert Jeu-Liang Sheu, The structure of twisted SU(3) groups $\ldots . \ldots \ldots . \ldots 307$

Morwen Thistlethwaite, On the algebraic part of an alternating link . . . . . 317

Thomas (Toma) V. Tonev, Multi-tuple hulls .................. 335

Arno van den Essen, A note on Meisters and Olech's proof of the global asymptotic stability Jacobian conjecture ..................... 351

Hendrik J. van Maldeghem, A characterization of the finite Moufang hexagons by generalized homologies

Bun Wong, A note on homotopy complex surfaces with negative tangent bundles

Chung-Tao Yang, Any Blaschke manifold of the homotopy type of $\mathbf{C} P^{n}$ has the right volume 